\title{
Clinico-Radiological Characteristics and Pathological Diagnosis of Cerebral Amyloid Angiopathy-Related Intracerebral Hemorrhage
}

\author{
Tadashi Doden, $\mathrm{MD},{ }^{*}+\ddagger$ Hiromasa Sato, $\mathrm{MD},{ }^{*}$ Etsuko Sasahara, MD, $\mathrm{PhD},{ }^{*}$ \\ Takahiro Murata, MD, PhD,§ Takehiro Yako, MD,§ Kazuo Kitazawa, MD, PhD, § \\ Kayoko Higuchi, MD, PhD, || Shigeaki Kobayashi, MD, PhD, $§$ and \\ Takao Hashimoto, MD, $\mathrm{PhD}^{*}+$
}

\begin{abstract}
Objective: We aim to clarify the clinico-radiological characteristics of cerebral amyloid angiopathy-related intracerebral hemorrhage and to investigate the efficacy of pathological diagnosis using biopsy specimens. Method: We retrospectively reviewed 253 consecutive patients with cortico-subcortical hemorrhage who had been admitted to Aizawa Hospital between January 2006 and July 2013. We had performed craniotomy and hematoma evacuation in 48 patients, as well as biopsy of the evacuated hematoma, cerebral parenchyma adjacent to the hematoma, or both, and they were classified according to the histological results (positive or negative for vascular amyloid deposition) and to the Boston criteria. We compared the clinicoradiological characteristics of cerebral amyloid angiopathy-related intracerebral hemorrhage. We also investigated the detection rate of cerebral amyloid angiopathy with respect to the origins of the specimens. Results: Pathological examination revealed that 22 subjects were positive for vascular amyloid. The number of the cerebral microbleeds located in the deep or infratentorial region was significantly larger in the negative group than in the positive group $(P<.05)$. There was no significant difference in the distribution of lobar cerebral microbleeds and in the prevalence of hypertension. In the probable cerebral amyloid angiopathyrelated intracerebral hemorrhage patients, the probability of having vascular amyloid detected by biopsy of both hematoma and parenchyma was $100 \%$. Rebleeding in the postoperative periods was observed in 2 cases $(9.1 \%)$ of the positive group. Conclusions: Our results demonstrate the importance and safety of biopsy simultaneously performed with hematoma evacuation. Deep or infratentorial microbleeds are less correlated with cerebral amyloid angiopathy-related intracerebral hemorrhage than with noncerebral amyloid angiopathy-related intracerebral hemorrhage.
\end{abstract}

\footnotetext{
From the *Department of Neurology, Aizawa Hospital, Matsumoto, Japan; +Brain Imaging Research Center, Aizawa Hospital, Matsumoto, Japan; łDepartment of Medicine (Neurology and Rheumatology), Shinshu University School of Medicine, Matsumoto, Japan; §Department of Neurosurgery, Aizawa Hospital, Matsumoto, Japan; and \|Department of Anatomic Pathology, Aizawa Hospital, Matsumoto, Japan. Received February 9, 2016; revision received March 15, 2016; accepted March 20, 2016.

Authors' roles: Tadashi Doden-patient evaluation, pathological evaluation, statistical analysis; wrote all drafts of manuscript. Hiromasa Sato-patient evaluation, interpretation of data. Etsuko Sasahara-interpretation of data. Takahiro Murata-patient evaluation, surgical intervention. Takehiro Yako-patient evaluation, surgical intervention. Kazuo Kitazawa-patient evaluation, surgical intervention. Kayoko Higuchi-pathological evaluation. Shigeaki Kobayashi—study design. Takao Hashimoto-study design, patient evaluation; edited drafts of manuscript.

Address correspondence to Tadashi Doden, MD, Department of Neurology, Aizawa Hospital, 2-5-1 Honjo, Matsumoto 390-8510, Japan. E-mail: aidr214@ai-hosp.or.jp.

$1052-3057 / \$$ - see front matter

(C) 2016 National Stroke Association. Published by Elsevier Inc. All rights reserved.

http:/ / dx.doi.org/10.1016/j.jstrokecerebrovasdis.2016.03.041
} 
Key Words: Cerebral amyloid angiopathy—intracerebral hemorrhage—cerebral microbleeds-biopsy—diagnosis.

(c) 2016 National Stroke Association. Published by Elsevier Inc. All rights reserved.

\section{Introduction}

Cerebral amyloid angiopathy (CAA) is caused by the accumulation of amyloid fibril on the cortical and leptomeningeal vessel walls, and it is an important cause of cerebral cortico-subcortical hemorrhage, cerebral infarction, leukoencephalopathy, cerebral vasculitis, and dementia in the elderly. ${ }^{1-4}$ The incidence of CAA increases with age. Approximately half of elderly people (aged 60 years and older) and 74\% of the individuals aged 90 years and older are affected with CAA. ${ }^{4} \mathrm{CAA}$ is closely related to the etiology of Alzheimer's disease (AD) and vascular dementia. CAA is commonly found with an incidence of about $80 \%-100 \%$ in AD. ${ }^{4-6}$ CAA leads to multiple cortico-subcortical or lobar hemorrhages in the elderly, and it is then referred to as CAA-related intracerebral hemorrhage (CAA-ICH). ${ }^{3,7}$ Boston criteria were established for CAA-ICH by the Boston Cerebral Amyloid Angiopathy Group, and a definite diagnosis of CAA$\mathrm{ICH}$ can be formulated only by demonstrating lobar or cortico-subcortical hemorrhage and severe CAA with vasculopathy after whole histological investigation of affected brain tissue is obtained at autopsy. ${ }^{8,9}$ Biopsy of the evacuated hematoma or cerebral cortex contributes to premortem diagnosis of probable CAA-ICH with supporting pathology; however, the positive ratio of amyloid deposition in the specimens obtained from brain biopsy or hematoma evacuation has not been investigated enough. ${ }^{9-12}$ We retrospectively searched the patients with clinically diagnosed CAA-ICH who underwent biopsy of evacuated hematoma, cerebral parenchyma, or both, and classified them into CAA-pathology positive and negative groups, depending on the pathological results. As a prerequisite, the CAA-pathology positive group could be estimated to have a higher ratio of definite CAA-ICH and a lower ratio of hypertensive $\mathrm{ICH}$ than the CAApathology negative group. We investigated the differences of clinico-radiological characteristics in these 2 groups. We also investigated the differences of positive ratios of amyloid deposition, depending on the site of biopsy.

\section{Methods}

We retrospectively reviewed 253 consecutive patients with cortico-subcortical hemorrhage who had been admitted to Aizawa Hospital Stroke Center between January 2006 and July 2013 (Fig 1, A). We had performed craniotomy and hematoma evacuation in 48 patients, and biopsy of evacuated hematoma, cerebral parenchyma adjacent to the hematoma, or both in 44 of these 48 patients. We divided 22 CAA-pathology positive patients and 22 CAApathology negative patients into 3 groups, "probable CAA," "possible CAA," and "excluded," respectively, according to the Boston criteria. The origins of the biopsy specimens in probable or possible CAA patients were evacuated hematoma in 6 patients, cerebral parenchyma in 13 patients, and both in 12 patients. This study was approved by the institutional ethical committee, and written informed consents for surgical treatment and biopsy were obtained from either the patients or their families.

In clinical and radiological evaluation, the age at onset, the clinical diagnosis of CAA-ICH (possible or probable), hypertension (blood pressure $\geqq 140 / 90 \mathrm{mmHg}$ ) before onset, antithrombotic drugs, dementia (Mini-Mental State Examination $\leqq 23$ ) before onset, estimated volume in hematoma, rebleeding, cerebral microbleeds (MBs), intraventricular hemorrhage (IVH), focal subarachnoid hemorrhage (SAH), cortical superficial siderosis (SS), and white matter lesions were compared between the CAApathology positive group and the CAA-pathology negative group. Brain computed tomography (CT) was performed in all patients, and volume in hematoma was estimated by the ABC/2 formula. ${ }^{13}$ Brain magnetic resonance imaging (MRI) was performed in 17 of the 22 CAA-pathology positive patients and in 7 of the 9 CAApathology negative patients. The ratio of rebleeding was calculated for all patients except for 2 CAA-pathology positive patients with whom contact had been lost. We checked for a history of antithrombotic drugs, hypertension, and dementia based on the information from patients' families and medical records.

The MRI techniques were as follows. Standardized T2weighted image (WI), fluid-attenuated inversion recovery, and $\mathrm{T}^{*}$ gradient-echo sequence $\left(\mathrm{T} 2{ }^{*} \mathrm{WI}\right)$ were acquired from 24 subjects using the 1.5 T MRI scanner (MAGNETOM Avanto, syngo MR VB17; Siemens, München, Germany). T2*WI was obtained using the following parameters: axial slice thickness $=5.0 \mathrm{~mm}$, interslice thickness $=1.5 \mathrm{~mm}$, repetition time $=737 \mathrm{~ms}$, echo time $=26 \mathrm{~ms}$, flip angle $=20^{\circ}$, and matrix size $=512 \times 512$ pixels.

MBs and cortical SS were evaluated in T2*WI. MBs are defined as focal areas of very low signal intensity, homogeneous round lesions with a diameter of $2-5 \mathrm{~mm}$; superficial vessels and small calcification in basal ganglia or dentate nucleus were excluded. ${ }^{14}$ Cortical SS is defined based on rims of hypointensity enveloping the surface of the cortical fissures. White matter lesions in the periventricular areas and deep or subcortical areas were graded into 4 stages, according to the method of the previous reports. ${ }^{15,16}$ Imaging analysis was done by an 
experienced neuroradiologist who had not been informed about the patients' clinical information.

For pathological investigation, all samples were stained by hematoxylin and eosin and by Congo red (Wako Pure Chemical Industries, Ltd., Osaka, Japan). Immunohistochemistry with antibody to $\beta$-amyloid $(A \beta)$ was also performed. $A \beta$ deposition in vascular walls was checked and CAA-pathology positive was diagnosed by circumferential deposition of $A \beta$. We investigated the CAA detection rate with respect to the origins of the specimens.

Statistical analysis was performed using chi-square test or Fisher exact test for categorical variates (chi-square test: white matter lesion; Fisher exact test: male sex, preoperative diagnosis, hypertension, dementia, antithrombotic drug, IVH, focal SAH, cortical SS, recurrence of hemorrhage, MBs) to compare the difference in the data between the 2 groups: the CAA-pathology positive group and the CAA-pathology negative group. Unpaired $t$-test or MannWhitney $U$-test was used for continuous variates (unpaired $t$-test: age, hematoma volume; Mann-Whitney $U$-test: total number of MBs in each patient, number of MBs). A twotailed $P$ value of $<.05$ was considered statistically significant. The statistical package (SPSS version 18.0 software, SPSS Inc., Chicago, IL, USA) was used for analyses.

\section{Results}

\section{Characteristics of the Study Groups}

The demographic characteristics of the patients are presented in Figure 1. Figure 1, B shows the clinical diagnoses of the 44 patients in the CAA-pathology positive and negative groups. Thirteen patients in the CAA-pathology negative group were excluded, according to the Boston criteria. Seven patients with probable CAA-ICH and 15 patients with possible CAA-ICH were identified in the CAA-pathology positive group. Two patients with probable CAA-ICH and 7 patients with possible CAA-ICH were identified in the CAA-pathology negative group.

\section{Comparison of the Clinical and Radiological Features between the CAA-Pathology Positive and CAA-Pathology Negative Groups}

Table 1 shows the clinico-radiological characteristics of the patients. There is no significant difference in the prevalence of hypertension and dementia between the CAApathology positive group and the CAA-pathology negative group. With regard to the difference in the distribution of MBs, the number of MBs located in cerebellum, brain stem, thalamus, and basal ganglia (deep or infratentorial MBs) was significantly larger in the CAA-pathology negative group than in the CAA-pathology positive group $(P<.05)$.

\section{Representative Cases}

Figure 2 shows the radiological and pathological features of the 3 representative cases with CAA-pathology positive and negative. A 68-year-old woman was pathologically diagnosed with CAA-pathology positive by biopsy of evacuated hematoma. Photomicrographs of evacuated hematoma demonstrated amyloid-laden blood vessels as concomitants of the arachnoidal tissue (Fig 2, A-F).

A 60-year-old woman with multiple subcortical hemorrhages underwent cortical biopsy of the left temporal lobe. Photomicrographs of biopsied cortical and meningeal tissue demonstrated amyloid-laden blood vessels (Fig 2, G-L).

An 82-year-old woman with multiple deep or infratentorial MBs underwent biopsy of the cerebral parenchyma adjacent to the hematoma, but CAA pathology was not detected (Fig 2, M-O).

Figure 3 shows the relationship between the sites of the biopsy specimen and the CAA pathology. It reveals that the highest ratio of CAA-pathology positive was found in the patients who had undergone biopsy from both hematoma and parenchyma.

Table 2 shows the results of CAA pathology in 12 patients who underwent biopsy from both hematoma and parenchyma. Five cases showed CAA-pathology positive in both hematoma and parenchyma. On the other hand, 1 case showed CAA-pathology positive only in hematoma. The CAA detection rate was $50.0 \%$ in hematoma and $83.3 \%$ in parenchyma. One patient who underwent biopsy of the cerebral cortex and hematoma in the left parietal lobe showed CAA-pathology negative. MBs of the patient were strictly distributed within the deep or infratentorial area.

Table 3 shows the relationship between the clinical diagnosis, the kind of biopsy specimen, and the probability of definitive CAA. We defined "definitive" CAA as clinically probable or possible CAA-ICH patients with pathological proof of vascular amyloid deposition. The probability of definitive CAA in patients who underwent biopsy of hematoma or parenchyma was $60 \%$ in probable CAA patients. On the other hand, all 4 of the patients who underwent biopsy of both hematoma and parenchyma were CAA-pathology positive; thus, the probability of definitive CAA was $100 \%$. In possible CAA patients, the probability of definitive CAA in patients who underwent biopsy of hematoma or parenchyma was $57 \%$. Two patients were clinically diagnosed as probable CAA but pathologically diagnosed as CAA-pathology negative. The vascular structure was not included in the evacuated hematoma in 1 patient and the cerebral white matter adjacent to the bottom (deep white matter, thus, not cerebral cortex) of the hematoma cavity was biopsied in the other patient.

\section{Rebleeding in the CAA-Pathology Positive Group}

The surgery of hematoma removal was performed from 5 hours to 22 days, mean 2.8 days, after ICH onset in our series. 
A

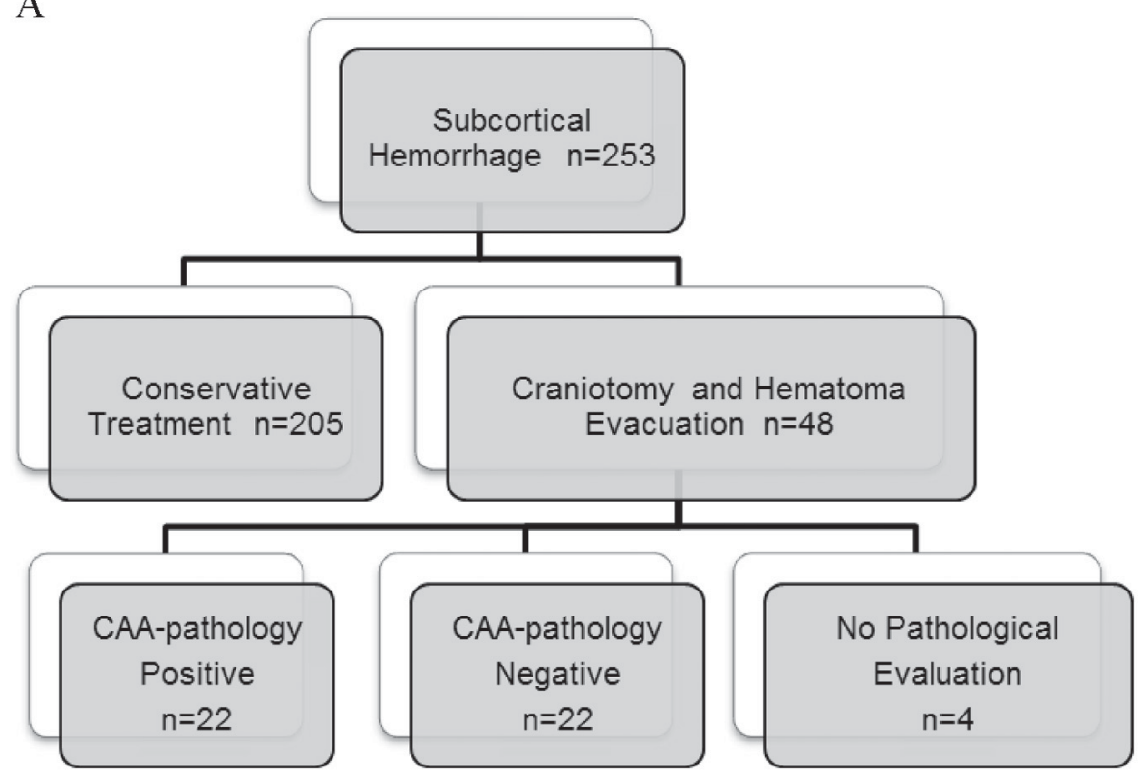

B

Clinical Diagnosis in

Patients with CAA-pathology positive

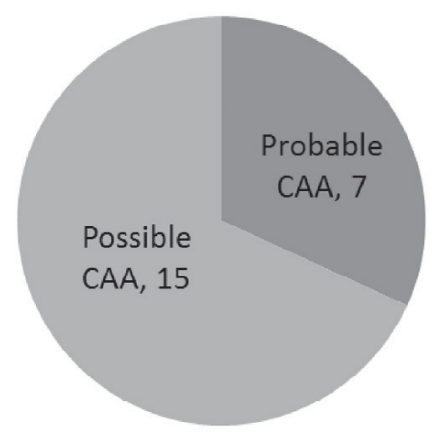

$\mathrm{n}=22$
Clinical Diagnosis in

Patients with CAA-pathology

negative

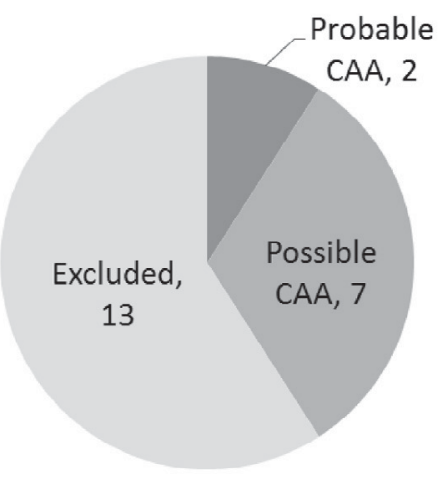

$\mathrm{n}=22$
Details of Excluded Patients

$\begin{array}{lr}\text { Age }<55 \text { years } & 2 \\ \text { Cerebral infarction } & 2 \\ \text { Brain tumor } & 1 \\ \text { Head trauma } & 1 \\ \text { Sinus thrombosis } & 1 \\ \text { Arteriovenous } & 1 \\ \text { malformation } & 1 \\ \text { Cavernous angioma } & 2 \\ \text { Thrombocytopenia } & 2 \\ \text { Coagulopathy } & \mathrm{n}=13 \\ \text { Total } & \end{array}$

Figure 1. Demographic characteristics of the patients. (A) Classification of the subjects. (B) Clinical diagnosis in patients with CAA-pathology positive group and negative group. Abbreviation: CAA, cerebral amyloid angiopathy.

Rebleeding in the postoperative periods was observed in 2 cases $(9.1 \%)$ of the CAA-pathology positive group. These 2 cases (a 70-year-old man and an 85-yearold woman) each presented a clinically severe condition due to a large hematoma (estimated volume in the hematoma was more than $75 \mathrm{ml}$ ) and needed a craniotomy and hematoma evacuation as critical care. Both of them died because of rebleeding associated with the initial surgical sites in the acute or subacute postoperative periods.

\section{Discussion}

Clinico-Radiological Characteristics of CAA-ICH

\section{Differences in the Distribution of MBs in CAA and Hypertensive Microangiopathy}

There was no significant difference in the number of lobar MBs between the CAA-pathology positive group and the CAA-pathology negative group, whereas the 
Table 1. Characteristics of the patients

\begin{tabular}{|c|c|c|c|c|c|}
\hline \multirow[b]{3}{*}{ Characteristics of the patients } & \multirow{2}{*}{\multicolumn{2}{|c|}{$\begin{array}{c}\text { CAA-pathology positive } \\
\mathrm{n}=22\end{array}$}} & \multirow{2}{*}{\multicolumn{2}{|c|}{$\begin{array}{c}\text { CAA-pathology negative } \\
\mathrm{n}=9\end{array}$}} & \multirow[b]{3}{*}{$P$} \\
\hline & & & & & \\
\hline & $\mathrm{n}$ & $\%$ & $\mathrm{n}$ & $\%$ & \\
\hline Age (years) & \multicolumn{2}{|c|}{$75.3 \pm 7.7$} & \multicolumn{2}{|c|}{$72.8 \pm 9.2$} & .447 \\
\hline Male sex & 6 & 27.3 & 4 & 44.4 & .302 \\
\hline \multicolumn{6}{|l|}{ Preoperative diagnosis } \\
\hline Probable CAA-ICH & 7 & 31.8 & 2 & 22.2 & .472 \\
\hline Possible CAA-ICH & 15 & 68.2 & 7 & 77.8 & \\
\hline Hypertension & 12 & 54.5 & 5 & 55.5 & .637 \\
\hline Dementia & 5 & 22.7 & 3 & 33.3 & .424 \\
\hline Antithrombotic drug & 4 & 18.2 & 1 & 11.1 & .595 \\
\hline Hematoma volume (ml) & \multicolumn{2}{|c|}{$69.6 \pm 36.2$} & \multicolumn{2}{|c|}{$58.8 \pm 31.5$} & .439 \\
\hline Intraventricular hemorrhage & 14 & 63.6 & 4 & 44.4 & .279 \\
\hline Focal subarachnoid hemorrhage & 11 & 50 & 2 & 22.2 & .154 \\
\hline Cortical superficial siderosis & 6 & 35.3 & 0 & 0 & .092 \\
\hline Recurrence of hemorrhage & 7 & 35 & 0 & 0 & .050 \\
\hline MBs & 11 & 64.7 & 6 & 85.7 & .306 \\
\hline Total number of MBs in each patient & & & & & .098 \\
\hline \multicolumn{6}{|l|}{ Number of MBs } \\
\hline Frontal lobe & \multicolumn{2}{|c|}{$.94 \pm 2.22$} & \multicolumn{2}{|c|}{$.14 \pm .38$} & .531 \\
\hline Parietal lobe & \multicolumn{2}{|c|}{$1.59 \pm 2.92$} & \multicolumn{2}{|c|}{0} & .079 \\
\hline Temporal lobe & \multicolumn{2}{|c|}{$.35 \pm .79$} & \multicolumn{2}{|c|}{$.14 \pm .38$} & .590 \\
\hline Occipital lobe & \multicolumn{2}{|c|}{$.47 \pm 1.38$} & \multicolumn{2}{|c|}{$.14 \pm .38$} & .956 \\
\hline Basal ganglia and thalamus & \multicolumn{2}{|c|}{$.06 \pm .24$} & \multicolumn{2}{|c|}{$1.71 \pm 1.50$} & $.001 *$ \\
\hline Brain stem & \multicolumn{2}{|c|}{$.18 \pm .53$} & \multicolumn{2}{|c|}{$1.57 \pm 1.90$} & $.015 *$ \\
\hline Cerebellum & \multicolumn{2}{|c|}{0} & \multicolumn{2}{|c|}{$1.71 \pm 2.56$} & $.001 *$ \\
\hline \multicolumn{6}{|l|}{ PVH grade } \\
\hline $0 ;$ none & 0 & 0 & 0 & 0 & .427 \\
\hline 1 ; caps or lining & 9 & 40.9 & 6 & 66.7 & \\
\hline 2 ; bands & 9 & 40.9 & 2 & 22.2 & \\
\hline 3 ; irregular & 4 & 18.2 & 1 & 11.1 & \\
\hline \multicolumn{6}{|l|}{ DSWMH grade } \\
\hline 0 ; none & 4 & 18.2 & 1 & 11.1 & .311 \\
\hline 1 ; punctate & 9 & 40.9 & 5 & 55.5 & \\
\hline 2 ; early confluent & 9 & 40.9 & 2 & 22.2 & \\
\hline 3 ; confluent & 0 & 0 & 1 & 11.1 & \\
\hline
\end{tabular}

Abbreviations: CAA, cerebral amyloid angiopathy; CAA-ICH, cerebral amyloid angiopathy-related intracerebral hemorrhage; DSWMH, deep and subcortical white matter hyperintensity; MBs, cerebral microbleeds; PVH, periventricular hyperintensity.

Chi-square test, Fisher exact test, $t$-test, or Mann-Whitney $U$-test was conducted.

$* P<.05$.

Figure 2. Brain images and photomicrographs of evacuated hematoma and cerebral cortex in three patients. The clinical diagnosis was probable CAA$I C H$ in the first 2 cases and possible CAA-ICH in the latter, according to the Boston criteria. (A-F) A 68-year-old woman. The CT revealed subcortical hemorrhage in the right frontal lobe $(A)$. There were no microbleeds in the basal ganglia or thalamus, and small calcifications were identified in the bilateral globus pallidus in MRI T2*WI (B). But several cortico-subcortical microbleeds and superficial siderosis were identified in the bilateral hemispheres (C). Craniotomy and hematoma evacuation was performed and photomicrographs of the evacuated hematoma demonstrated amyloid-laden blood vessels as concomitants of the arachnoidal tissue ( $D$; hematoxylin and eosin, original magnification 200x, E; immunohistochemistry with antibody to $A \beta, F$; Congo red stain, upper-right side photomicrograph is under polarized light). (G-L) A 60-year-old woman. The brain CT and MRI T2*WI image revealed acute subcortical hemorrhages in the bilateral temporal lobes and an old subcortical hematoma in the left parietal lobe. There were multiple lobar microbleeds in the bilateral hemispheres $(G, H, I)$. Photomicrographs of biopsied cortical and meningeal tissue, demonstrating amyloid-laden blood vessels (J; hematoxylin and eosin stain, original magnification 200x, K; immunohistochemistry with antibody to AB, L; Congo red stain under polarized light). (M-O) An 82year-old woman. The brain CT revealed massive hematoma in the right temporo-parietal lobe $(M)$. T2*WI demonstrated multiple microbleeds in the brainstem, cerebellar hemisphere, and basal ganglia $(N, O)$. The woman underwent hematoma evacuation and biopsy from the cerebral parenchyma adjacent to the hematoma cavity. CAA pathology was not detected from these specimens. Abbreviations: A $\beta$, $\beta$-amyloid; $C A A-I C H$, cerebral amyloid angiopathy-related intracerebral hemorrhage; $C T$, computed tomography; MRI, magnetic resonance imaging; $T 2^{*} \mathrm{WI}, \mathrm{T} 2^{*}$-weighted image. 

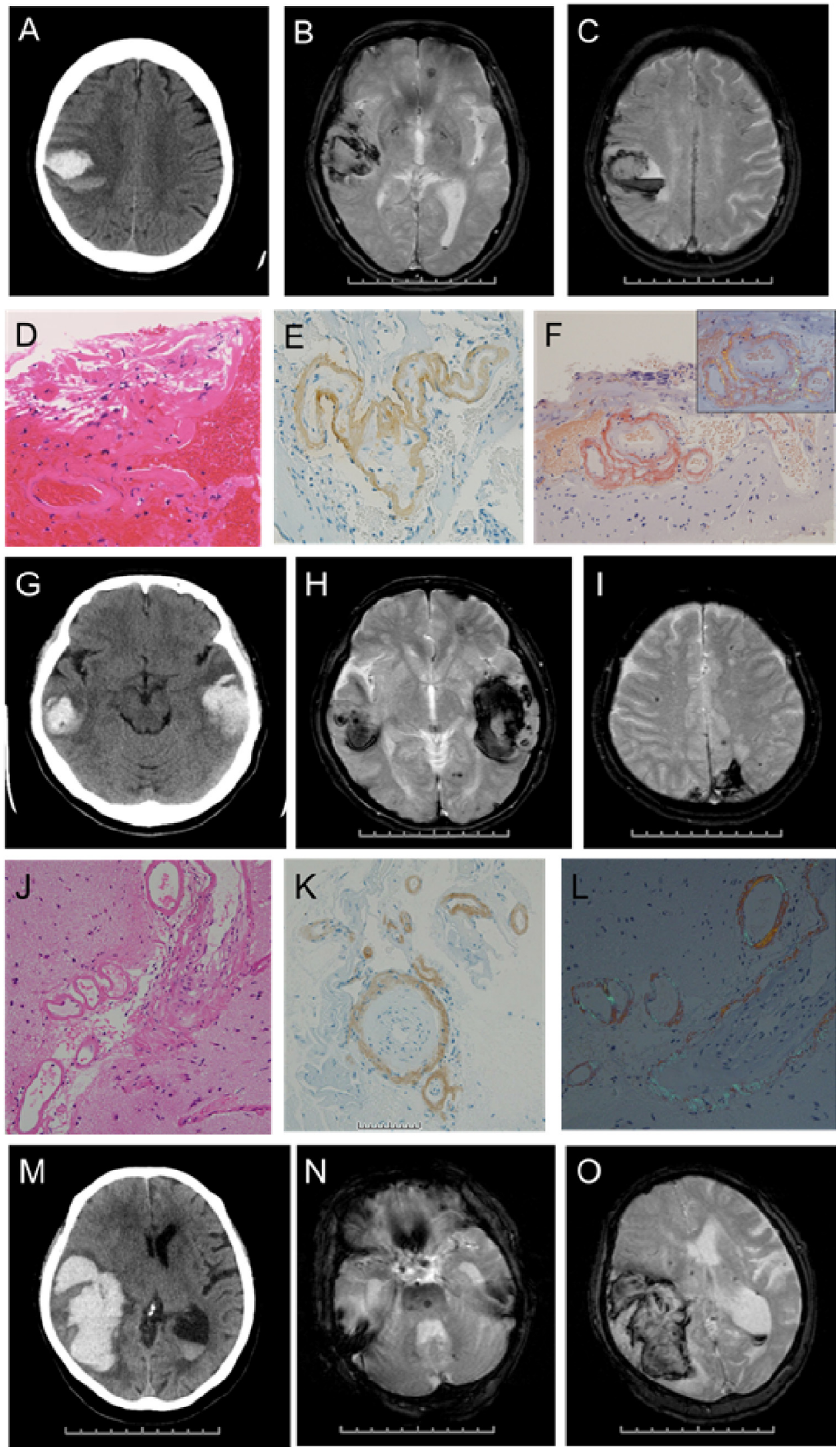
(n)

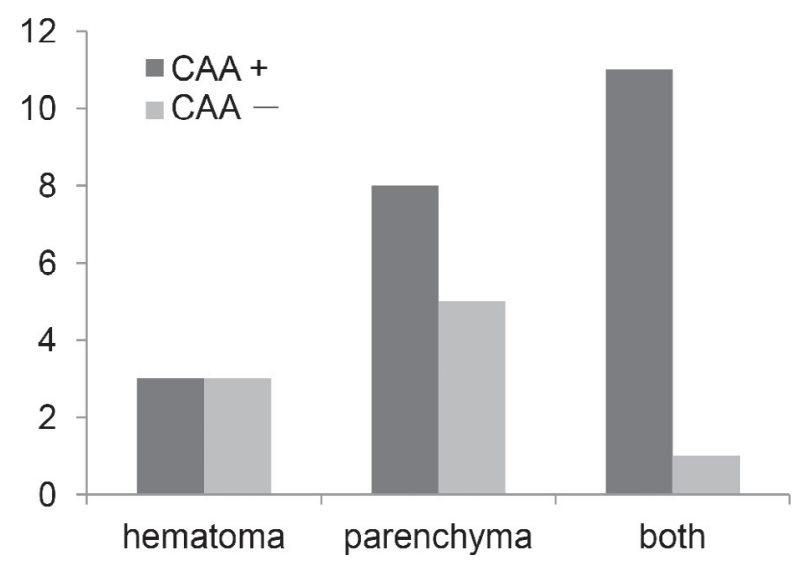

Figure 3. Details of tissue sample obtained by biopsy and CAA pathology. The ratio of CAA-pathology positive in each kind of specimen was $50 \%, 61.5 \%$, and $91.7 \%$ in hematoma, parenchyma, and both, respectively. Abbreviations: CAA, cerebral amyloid angiopathy; $\square C A A+; C A A-$.

number of deep or infratentorial MBs in the CAApathology positive group was significantly lower than in the CAA-pathology negative group. MBs represent hemosiderin-containing macrophages which have englobed red blood cells derived from extravasation from small vessels, and they are correlated with bleeding-

Table 2. Results of CAA pathology in 12 patients who underwent biopsy from both hematoma and parenchyma

Distribution of CAA pathology

\begin{tabular}{lc} 
Hematoma & 1 \\
Hematoma and parenchyma & 5 \\
Parenchyma & 5 \\
Total & $11^{*}$ \\
\hline
\end{tabular}

Abbreviation: CAA, cerebral amyloid angiopathy.

*One patient with CAA-pathology negative was excluded. prone microangiopathy such as lipohyalinosis or CAA. ${ }^{17}$ Cortico-subcortical areas are preferentially affected by MBs in accordance with the distribution of $\mathrm{A} \beta$ deposition in vascular walls in patients with CAA. ${ }^{17-19}$ Deep or infratentorial MBs were positively correlated with CAApathology negative in the present study. A previous study suggested that deep or infratentorial MBs are relevant to cardiovascular risk factors, the presence of lacunar infarcts, white matter lesions, and underlying hypertension..$^{20}$ Deep MBs have been suggested to be relevant to subcortical small vessel disease (SVD), such as lacunar infarction or white matter lesions, but not to amyloid burden. ${ }^{21}$ Thus, deep or infratentorial MBs strongly suggest the existence of hypertensive microangiopathy rather than CAA.

\section{Relationship between CAA-ICH and Hypertension}

In our study, there is no significant difference in prevalence of the hypertension between the CAA-pathology positive group and the CAA-pathology negative group. The progression of CAA causes fibrinoid necrosis and, as a result, microaneurysm formation, secondly to focal bulging of the affected vascular wall, could be observed. These structural changes of the affected vessels are important as contributing factors in the development of CAA-ICH and are collectively called CAAassociated vasculopathy. ${ }^{3}$ These pathological changes are also observed in patients with cerebral SVD resulting from arteriosclerotic diseases such as hypertension or diabetes mellitus. ${ }^{22}$

In the past, the development of CAA had not been thought to correlate with the presence of common cerebrovascular risk factors, including hypertension, diabetes mellitus, hyperlipidemia, or the severity of atherosclerosis of the cerebral arteries. ${ }^{23}$ Thereafter, the correlation between CAA, on the one hand, and cerebral arteriosclerosis and arteriolar sclerosis, on the other, has attracted attention. ${ }^{6}$ Recently, the correlation in the severity of SVD

Table 3. Relationship between clinical diagnosis, kind of specimen, and probability of definitive CAA

\begin{tabular}{|c|c|c|c|c|c|}
\hline \multirow[b]{2}{*}{ Clinical diagnosis } & \multirow[b]{2}{*}{ Specimen } & \multirow[b]{2}{*}{$\mathrm{n}$} & \multicolumn{2}{|c|}{ Pathological diagnosis } & \multirow[b]{2}{*}{ Probability of definitive CAA (\%) } \\
\hline & & & CAA-pathology positive & CAA-pathology negative & \\
\hline \multirow[t]{4}{*}{ Probable CAA } & All & 9 & 7 & 2 & 77.8 \\
\hline & Hematoma & 1 & 0 & 1 & .0 \\
\hline & Parenchyma & 4 & 3 & 1 & 75.0 \\
\hline & Both & 4 & 4 & 0 & 100.0 \\
\hline \multirow[t]{4}{*}{ Possible CAA } & All & 22 & 15 & 7 & 68.2 \\
\hline & Hematoma & 5 & 3 & 2 & 60.0 \\
\hline & Parenchyma & 9 & 5 & 4 & 55.6 \\
\hline & Both & 8 & 7 & 1 & 87.5 \\
\hline Total & & 31 & 22 & 9 & 71.0 \\
\hline
\end{tabular}

Abbreviation: CAA, cerebral amyloid angiopathy. 
and CAA was demonstrated by postmortem pathological investigation of elderly subjects..$^{5}$ As mentioned above, the prevalence of CAA in the elderly increases with age. An autopsy series reported that the prevalence of CAA over the age of 70 years was $43 \%$, and almost half of the cases were estimated to have moderate-to-severe CAA. ${ }^{3}$ The prevalence of hypertension also increases with the age. The prevalence of hypertension at age 50 is about $30 \%$ and $20 \%$ in the United States and Canada, respectively, and it increases to about $60 \%$ and $50 \%$ at age $70 .{ }^{24}$ As the prevalence of CAA and hypertension increases in the elderly, it is probable that the rate of their coexistence is higher in older people. Actually, Smith et al. reported that $25 \%$ of patients with $\mathrm{ICH}$ had both CAA and hypertensive microangiopathy. ${ }^{25}$ It was pointed out that the coexistence of CAA and hypertension might be a cause of the wide distribution of MBs. ${ }^{17}$ Thus, structural degenerations of the cortical and/or leptomeningeal vessel walls such as fibrinoid necrosis or microaneurysm formation exist in patients with CAA, and high blood pressure may trigger a rupture of the vessel walls, which results in lobar hemorrhage. We cannot exclude the possibility of CAA-ICH in the diagnosis of patients with lobar hemorrhage and hypertension, ${ }^{12}$ and it is difficult to strictly distinguish CAA-ICH from hypertensive ICH solely based on clinical and radiological findings. ${ }^{26}$

\section{Focal SAH and Cortical SS in the Diagnosis of CAA}

In this study, we investigated the relationship between CAA pathology and hemorrhagic lesions associated with ICH on MRI such as focal SAH or cortical SS. SAH and cortical SS tended to be observed more frequently in the CAA-pathology positive group. This result supports the hypothesis that various hemorrhagic lesions are elicited with the progression of CAA-associated vasculopathy. ${ }^{27,28}$

\section{Factors That Influence the Detection of CAA Pathology}

The highest ratio of CAA-pathology positive was obtained by biopsy of both hematoma and cerebral cortex in the present study. Furthermore, the biopsy of cerebral parenchyma showed a higher CAA detection rate than did the biopsy of hematoma in the 12 patients who underwent biopsy of both hematoma and cerebral parenchyma. Our results support the notion that histopathological examination of both cerebral cortex and hematoma is necessary to make a reliable diagnosis of CAA, as shown in the Boston criteria. ${ }^{8,9}$ However, CAA distribution is characteristically patchy and segmental; it may lead to the possibility of having false-negative cases. ${ }^{1,29}$

On the other hand, several attempts using amyloid imaging, such as ${ }^{11} \mathrm{C}$-Pittsburgh compound B positron emission tomography $\left({ }^{11} \mathrm{C}-\mathrm{PiB} \mathrm{PET}\right)$, have been made to detect CAA as an underlying disease in several pathological conditions such as dementia, cortical $\mathrm{SAH}$, and cerebral angitis. ${ }^{30-34}$ A positive relationship between local amyloid burden and a future risk for hemorrhage has been suggested based on the result of a longitudinal cohort study with ${ }^{11} \mathrm{C}-\mathrm{PiB}$ PET and MRI. ${ }^{31}$ However, the presence of vascular amyloid in the tissue sample is not perfectly specific for CAA-ICH because elderly people may incidentally present with asymptomatic vascular amyloid deposition. ${ }^{3,4,11}$ For more definitive diagnosis of CAA-ICH, ruptured amyloid-laden blood vessels adjacent to the hematoma should be identified in postmortem study. ${ }^{35}$

\section{Safety of Craniotomy and Biopsy in Patients with CAA-ICH}

The rebleeding ratio after craniotomy and hematoma evacuation in patients with CAA-pathology positive is $9.1 \%$ ( 2 of 22 cases) in our patients, and both patients died from rebleeding. On the other hand, no apparent adverse events related to surgical interventions were observed in the other patients. Previous studies have indicated that neurosurgical procedures in patients with CAA may cause uncontrollable perioperative and postoperative hemorrhages, and they have not recommended surgical interventions. ${ }^{36-38}$ On the other hand, the safety and effectiveness of hematoma evacuation for patients with CAA$\mathrm{ICH}$ have been warranted in recent years. ${ }^{39,40}$ Several reports have also referred to the safety of the biopsy of the cerebral cortex in patients with CAA-ICH. ${ }^{41-44}$ Mehndiratta et al. reported that the probability of postoperative hemorrhage within the first 48 hours of surgery was $11.8 \%$ on average (the probability ranged from $0 \%$ to $22 \%$ among the previous reports), and the mortality rate was $24.4 \%$ on average $(0 \%-75 \%)$ in patients with CAA-ICH. The patients' poor outcome in CAA-ICH with surgical intervention was estimated to be associated with poor preoperative functional status, age over 75 years, preoperative diagnosis of dementia, hematoma volume greater than $60 \mathrm{ml}$, the presence of $\mathrm{IVH}$, and postoperative hemorrhage. ${ }^{45}$

The time to surgery after ICH onset is one of the critical factors affecting the rate of rebleeding. A previous report presented the rate of rebleeding as $12 \%$ of the patients, mostly with deep ICH, who had been treated by open craniotomy and hematoma evacuation within 12 hours. ${ }^{46}$ Another study reported that $3.8 \%$ of the patients, mostly with putaminal or thalamic hemorrhage, had rebleeding after CT-guided stereotactic surgery; $76 \%$ had been performed later than 3 days after symptom onset. ${ }^{47}$ The rate of rebleeding and the timing of surgery in our cases fell within the ranges of the results of these previous reports, supporting the relative safety of hematoma evacuation surgery in CAA-ICH. Early surgical treatment might have functional and survival advantage for the patients with spontaneous superficial ICH without $\mathrm{IVH}^{48-50}$; however, careful consideration of the indication for surgical intervention and perioperative management is still needed, especially for the patients at high risk of surgical intervention. 


\section{Limitations of This Study}

This is a clinical research study that was planned and executed in a single institution, and the sample size is relatively small. Standardization of the specimens and biopsy locations were not strictly controlled; the specimens were obtained from cerebral white matter, not cerebral cortex in a few cases; and the evacuated hematoma contained no vessel. Furthermore, the distribution of CAA is patchy and segmental. Therefore, there could be falsenegative cases in the CAA-pathology negative group. Postmortem examination is essential for the definite pathological diagnosis of CAA at the present time, and a largescale comparative study that deals with clinical diagnosis based on the clinico-radiological findings, including amyloid imaging and pathological diagnosis obtained from biopsy and autopsy, is needed.

\section{Conclusion}

Deep or infratentorial MBs in $\mathrm{T} 2{ }^{*} \mathrm{WI}$ are less correlated with CAA-ICH than with non-CAA-ICH.

Hypertension may play an important role in the pathogenesis of CAA-ICH.

With regard to biopsy and pathological diagnosis of CAA-ICH performed with craniotomy and hematoma evacuation, the amyloid detection rate of biopsy of hematoma alone was low compared with the rate of biopsy of both hematoma and cerebral parenchyma. A specimen of the cerebral cortex adjacent to the hematoma should be obtained to detect CAA.

Hematoma evacuation surgery for CAA-ICH is relatively safe, but it should be carefully performed.

Acknowledgment: This study was supported in part by the technicians in Aizawa Hospital Department of Anatomic Pathology. The authors are grateful to the technicians for their excellent work.

\section{References}

1. Biffi A, Greenberg SM. Cerebral amyloid angiopathy: a systematic review. J Clin Neurol 2011;7:1-9.

2. Greenberg SM, Gurol ME, Rosand J, et al. Amyloid angiopathy-related vascular cognitive impairment. Stroke 2004;35:2616-2619.

3. Vinters HV. Cerebral amyloid angiopathy. A critical review. Stroke 1987;18:311-324.

4. Yamada M. Cerebral amyloid angiopathy: an overview. Neuropathology 2000;20:8-22.

5. Grinberg LT, Thal DR. Vascular pathology in the aged human brain. Acta Neuropathol 2010;119:277-290.

6. Ellis RJ, Olichney JM, Thal LJ, et al. Cerebral amyloid angiopathy in the brains of patients with Alzheimer's disease: the CERAD experience, Part XV. Neurology 1996;46:1592-1596.

7. Kase CS, Caplan LR. Lobar hemorrhage. Boston: Butterworth-Heinemann, 1994.

8. Greenberg SM, Briggs ME, Hyman BT, et al. Apolipoprotein E epsilon 4 is associated with the presence and earlier onset of hemorrhage in cerebral amyloid angiopathy. Stroke 1996;27:1333-1337.

9. Knudsen KA, Rosand J, Karluk D, et al. Clinical diagnosis of cerebral amyloid angiopathy: validation of the Boston criteria. Neurology 2001;56:537-539.

10. McCarron MO, Nicoll JA, Love S, et al. Surgical intervention, biopsy and APOE genotype in cerebral amyloid angiopathy-related haemorrhage. Br J Neurosurg 1999;13:462-467.

11. Greenberg SM, Vonsattel JP. Diagnosis of cerebral amyloid angiopathy. Sensitivity and specificity of cortical biopsy. Stroke 1997:28:1418-1422.

12. Smith EE, Greenberg SM. Clinical diagnosis of cerebral amyloid angiopathy: validation of the Boston criteria. Curr Atheroscler Rep 2003;5:260-266.

13. Kothari RU, Brott T, Broderick JP, et al. The ABCs of measuring intracerebral hemorrhage volumes. Stroke 1996;27:1304-1305.

14. Roob G, Lechner A, Schmidt R, et al. Frequency and location of microbleeds in patients with primary intracerebral hemorrhage. Stroke 2000;31:2665-2669.

15. Fazekas F, Niederkorn K, Schmidt R, et al. White matter signal abnormalities in normal individuals: correlation with carotid ultrasonography, cerebral blood flow measurements, and cerebrovascular risk factors. Stroke 1988;19:1285-1288.

16. Kapeller P, Barber R, Vermeulen RJ, et al. Visual rating of age-related white matter changes on magnetic resonance imaging: scale comparison, interrater agreement, and correlations with quantitative measurements. Stroke 2003;34:441-445.

17. Fazekas F, Kleinert R, Roob G, et al. Histopathologic analysis of foci of signal loss on gradient-echo $\mathrm{T} 2{ }^{*}$ weighted MR images in patients with spontaneous intracerebral hemorrhage: evidence of microangiopathyrelated microbleeds. AJNR Am J Neuroradiol 1999;20:637642.

18. Koennecke HC. Cerebral microbleeds on MRI: prevalence, associations, and potential clinical implications. Neurology 2006;66:165-171.

19. Vernooij MW, van der Lugt A, Ikram MA, et al. Prevalence and risk factors of cerebral microbleeds: the Rotterdam Scan Study. Neurology 2008;70:12081214.

20. Rosand J. Hypertension and the brain: stroke is just the tip of the iceberg. Neurology 2004;63:6-7.

21. Park JH, Seo SW, Kim C, et al. Pathogenesis of cerebral microbleeds: in vivo imaging of amyloid and subcortical ischemic small vessel disease in 226 individuals with cognitive impairment. Ann Neurol 2013;73:584-593.

22. Lammie GA, Brannan F, Slattery J, et al. Nonhypertensive cerebral small-vessel disease. An autopsy study. Stroke 1997;28:2222-2229.

23. Yamada M, Tsukagoshi H, Otomo E, et al. Cerebral amyloid angiopathy in the aged. J Neurol 1987;234:371376.

24. Bolivar JJ. Essential hypertension: an approach to its etiology and neurogenic pathophysiology. Int J Hypertens 2013;2013:547809.

25. Smith EE, Nandigam KR, Chen YW, et al. MRI markers of small vessel disease in lobar and deep hemispheric intracerebral hemorrhage. Stroke 2010;41:1933-1938.

26. Greenberg SM, Edgar MA. Case records of the Massachusetts General Hospital. Weekly clinicopathological exercises. Case 22-1996. Cerebral hemorrhage in a 69-year-old woman receiving warfarin. N Engl J Med 1996;335:189-196. 
27. Linn J, Halpin A, Demaerel P, et al. Prevalence of superficial siderosis in patients with cerebral amyloid angiopathy. Neurology 2010;74:1346-1350.

28. Yamada M. Cerebral amyloid angiopathy: emerging concepts. J Stroke 2015;17:17-30.

29. Vinters HV, Gilbert JJ. Cerebral amyloid angiopathy: incidence and complications in the aging brain. II. The distribution of amyloid vascular changes. Stroke 1983;14:924-928.

30. Baron JC, Farid K, Dolan E, et al. Diagnostic utility of amyloid PET in cerebral amyloid angiopathy-related symptomatic intracerebral hemorrhage. J Cereb Blood Flow Metab 2014;34:753-758.

31. Gurol ME, Dierksen G, Betensky R, et al. Predicting sites of new hemorrhage with amyloid imaging in cerebral amyloid angiopathy. Neurology 2012;79:320-326.

32. Gurol ME, Viswanathan A, Gidicsin C, et al. Cerebral amyloid angiopathy burden associated with leukoaraiosis: a positron emission tomography/magnetic resonance imaging study. Ann Neurol 2013;73:529-536.

33. Sengoku R, Matsushima S, Murakami Y, et al. (11)C PiB PET imaging of encephalopathy associated with cerebral amyloid angiopathy. Intern Med 2014;53:19972000.

34. Ly JV, Singhal S, Rowe CC, et al. Convexity subarachnoid hemorrhage with $\mathrm{PiB}$ positive pet scans: clinical features and prognosis. J Neuroimaging 2015;25:420-429.

35. Takeda S, Yamazaki K, Miyakawa T, et al. Subcortical hematoma caused by cerebral amyloid angiopathy: does the first evidence of hemorrhage occur in the subarachnoid space? Neuropathology 2003;23:254-261.

36. Kalyan-Raman UP, Kalyan-Raman K. Cerebral amyloid angiopathy causing intracranial hemorrhage. Ann Neurol $1984 ; 16: 321-329$

37. Ishii N, Nishihara Y, Horie A. Amyloid angiopathy and lobar cerebral haemorrhage. J Neurol Neurosurg Psychiatry 1984;47:1203-1210.

38. Torack RM. Congophilic angiopathy complicated by surgery and massive hemorrhage. A light and electron microscopic study. Am J Pathol 1975;81:349-366.

39. Izumihara A, Ishihara $\mathrm{T}$, Iwamoto $\mathrm{N}$, et al. Postoperative outcome of 37 patients with lobar intracerebral hemorrhage related to cerebral amyloid angiopathy. Stroke 1999;30:29-33.

40. Zhang Y, Wang X, Schultz C, et al. Postoperative outcome of cerebral amyloid angiopathy-related lobar intracerebral hemorrhage: case series and systematic review. Neurosurgery 2012;70:125-130, discussion 130 .

41. Matkovic Z, Davis S, Gonzales M, et al. Surgical risk of hemorrhage in cerebral amyloid angiopathy. Stroke 1991;22:456-461

42. Greene GM, Godersky JC, Biller J, et al. Surgical experience with cerebral amyloid angiopathy. Stroke 1990;21:1545-1549.

43. Minakawa T, Takeuchi S, Sasaki O, et al. Surgical experience with massive lobar haemorrhage caused by cerebral amyloid angiopathy. Acta Neurochir (Wien) 1995;132:48-52.

44. Leblanc R, Preul M, Robitaille Y, et al. Surgical considerations in cerebral amyloid angiopathy. Neurosurgery 1991;29:712-718.

45. Mehndiratta P, Manjila S, Ostergard T, et al. Cerebral amyloid angiopathy-associated intracerebral hemorrhage: pathology and management. Neurosurg Focus 2012;32:E7.

46. Morgenstern LB, Demchuk AM, Kim DH, et al. Rebleeding leads to poor outcome in ultra-early craniotomy for intracerebral hemorrhage. Neurology 2001;56:1294-1299.

47. Hokama M, Tanizaki Y, Mastuo K, et al. Indications and limitations for CT-guided stereotaxic surgery of hypertensive intracerebral haemorrhage, based on the analysis of postoperative complications and poor ability of daily living in 158 cases. Acta Neurochir (Wien) 1993;125:27-33.

48. Mendelow AD, Unterberg A. Surgical treatment of intracerebral haemorrhage. Curr Opin Crit Care 2007;13:169-174.

49. Qureshi AI, Mendelow AD, Hanley DF. Intracerebral haemorrhage. Lancet 2009;373:1632-1644.

50. Mendelow AD, Gregson BA, Rowan EN, et al. Early surgery versus initial conservative treatment in patients with spontaneous supratentorial lobar intracerebral haematomas (STICH II): a randomised trial. Lancet 2013;382:397-408 